\title{
Review
}

Harish C. Thakura, Madhurendra Singh, Luitgard Nagel-Steger, Daniel Prumbaum, Eyad Kalawy Fansa, Lothar Gremer, Hakima Ezzahoini, André Abts, Lutz Schmitt, Stefan Raunser, Mohammad R. Ahmadian and Roland P. Piekorz*

\section{Role of centrosomal adaptor proteins of the TACC family in the regulation of microtubule dynamics during mitotic cell division}

\begin{abstract}
During the mitotic division cycle, cells pass through an extensive microtubule rearrangement process where microtubules forming the mitotic spindle apparatus are dynamically instable. Several centrosomal- and microtubule-associated proteins are involved in the regulation of microtubule dynamics and stability during mitosis. Here, we focus on members of the transforming acidic coiled coil (TACC) family of centrosomal adaptor proteins, in particular TACC3, in which their subcellular localization at the mitotic spindle apparatus is controlled by Aurora-A kinase-mediated phosphorylation. At the effector level, several TACC-binding partners have been identified and characterized in greater detail, in particular, the microtubule polymerase XMAP215/ch-TOG/CKAP5 and clathrin heavy chain (CHC). We summarize the recent progress in the molecular understanding of these TACC3 protein complexes, which are crucial for proper mitotic spindle assembly and dynamics to prevent faulty cell division and aneuploidy. In this regard, the (patho)biological role of TACC3 in development and cancer will be discussed.
\end{abstract}

Keywords: cancer; centrosome; chTOG/CKAP5; microtubules; mitosis; TACC3.

\footnotetext{
aPresent address: Wellcome Trust Centre for Cell Biology, University of Edinburgh, Edinburgh, UK

*Corresponding author: Roland P. Piekorz, Institut für Biochemie und Molekularbiologie II, Medizinische Fakultät der Heinrich-HeineUniversität, D-40225 Düsseldorf, Germany, e-mail: Roland.Piekorz@uni-duesseldorf.de Harish C. Thakur, Madhurendra Singh, Eyad Kalawy Fansa, Hakima Ezzahoini and Mohammad R. Ahmadian: Institut für Biochemie und Molekularbiologie II, Medizinische Fakultät der Heinrich-Heine-Universität, D-40225 Düsseldorf, Germany Luitgard Nagel-Steger and Lothar Gremer: Institut für Physikalische Biologie, Heinrich-Heine-Universität, D-40225 Düsseldorf, Germany; and Strukturbiochemie (ICS-6), Forschungszentrum Jülich, Jülich, Germany
}

Daniel Prumbaum and Stefan Raunser: Max-Planck-Institut für Molekulare Physiologie, Dortmund, Germany André Abts and Lutz Schmitt: Institut für Biochemie, HeinrichHeine-Universität, D-40225 Düsseldorf, Germany

\section{Introduction}

Mitosis, the shortest phase within the cell cycle, is a fundamental cell division process that is essential for embryonic development and postnatal proliferative tissue homeostasis and function. Normal mitosis ensures an equal distribution of the genetic material duplicated during S-phase into two identical daughter cells (karyokinesis), which become finally separated during cytokinesis. Mitosis is a very complex and tightly controlled process where several biomolecules including numerous proteins and protein complexes are involved as important structural, functional, and regulatory elements. The mitotic spindle apparatus is comprised of centrosomes/ spindle poles, spindle microtubules, and microtubuleassociated protein complexes (Bornens, 2002, 2012); which are also involved in kinetochore protein interactions at the centromeric region of chromosomes. More than 200 proteins are responsible for spindle pole formation, chromosome alignment and segregation, cytokinesis during the cell division process (Andersen et al., 2003; Hubner et al., 2010; Hutchins et al., 2010; Neumann et al., 2010) (http://www.mitocheck.org/), as well as spindle checkpoint function that monitors correct microtubule-kinetochore attachment, thereby controlling metaphase to anaphase transition (Rieder and Maiato, 2004; Rieder, 2011; Khodjakov and Rieder, 2009; Musacchio, 2011; DeLuca and Musacchio, 2012).

Centrosomes are characteristic features of all metazoans functioning as microtubule organizing centers 
(MTOC) at spindle poles (Bornens, 2002, 2012; Azimzadeh and Bornens, 2005). Centrosomes are comprised of a perpendicularly aligned pair of centrioles surrounded by pericentriolar matrix (PCM) components. Microtubules as cytoskeletal elements of the spindle apparatus are hollow cylindrical structures, which are involved in the formation of cilia, flagella, axonemes, and spindle fibers (Nigg and Raff, 2009). In dividing cells, arrays of aster microtubules originate from MTOCs and connect them to the chromosomes at the metaphase plate (Compton, 2000). During the $\mathrm{G}_{2}-\mathrm{M}$-phase of the cell cycle, cells pass through a huge microtubule arrangement process that functions as an important regulatory switch and consists of microtubule nucleation, elongation, polymerization, and depolymerization (Mitchison and Kirschner, 1984; Desai and Mitchison, 1997). Aster microtubules are, in particular, highly dynamic structures, which are elongated by continuous addition of GTP bound $\alpha \beta$-tubulin heterodimers to the growing plus ends, where their depolymerization is triggered by hydrolysis of bound GTP to GDP and $\mathrm{P}_{\mathrm{i}}$ (Mitchison and Kirschner, 1984). Microtubules are dynamically unstable at their plus ends, which is characterized by two different transitions, called catastrophe (transition from polymerization to shrinkage) and rescue (transition from depolymerization to growth) (Figure 1) (Mitchison and Kirschner, 1984; Gardner et al., 2013). Therefore, microtubule length and stability depend on the shift of the equilibrium between these two transition states that is primarily regulated by several microtubule-associated proteins (MAPs).

Among the MAPs, the centrosomal transforming acidic coiled coil (TACC) protein family comprises important components of the mitotic spindle apparatus (Gergely et al., 2000a; Gergely, 2002; Raff, 2002; Peset and Vernos, 2008; Ha et al., 2013a). Vertebrates express three TACC isoforms, TACC1, TACC2, and TACC3 (Still et al., 1999a, 2004; Gomez-Baldo et al., 2010). As discussed below in greater detail, TACCs are regulated by mitotic kinases, in particular, Aurora-A, and function at the mitotic spindle apparatus as adaptor proteins, thereby, interacting, among others, with microtubule polymerases of the evolutionary conserved Xenopus microtubule-associated protein $215 \mathrm{kDa}$ (XMAP215)/minispindles (Msps)/colonic and hepatic tumor overexpressed gene (chTOG)/cytoskeleton-associated protein 5 (CKAP5) family. This effector complex plays a key role in the regulation of centrosomedependent assembly of spindle microtubules as well as their dynamics and stability during mitosis (Conte et al., 2003; Gergely et al., 2003; Peset et al., 2005; Peset and Vernos, 2008).

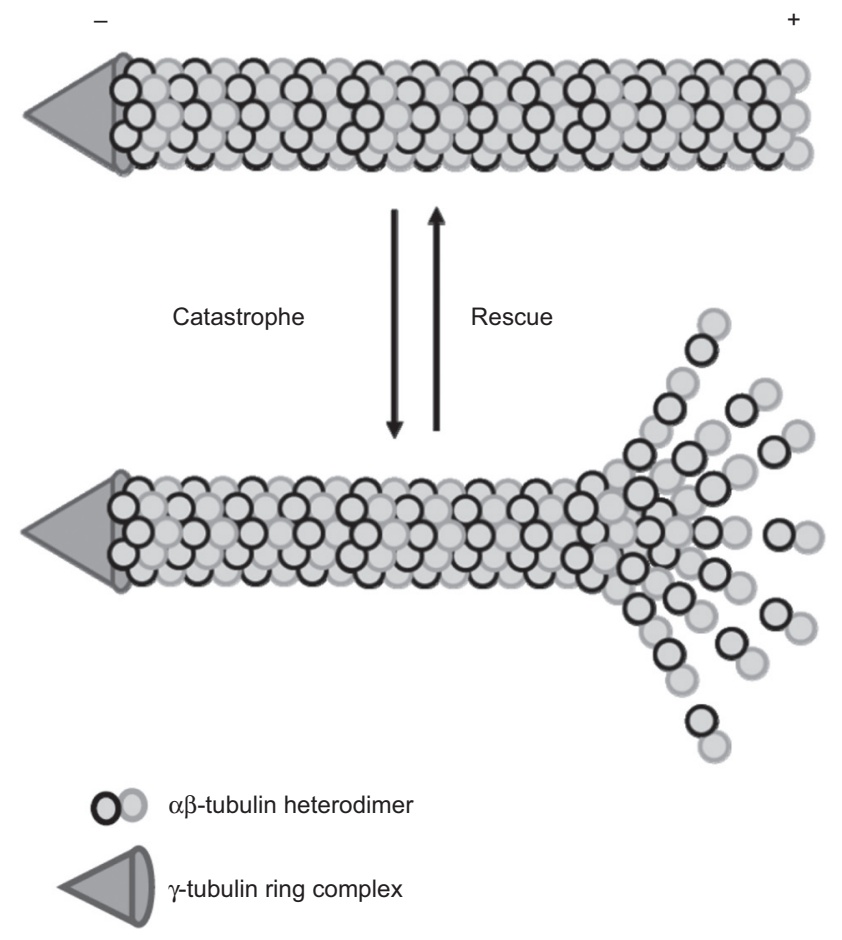

Figure 1 Microtubule dynamics during mitosis. The $\gamma$-tubulin ring complex primes microtubule nucleation and polymerization. Further elongation of microtubules is dependent on the shift in equilibrium between two transition states, catastrophe (polymerization $\rightarrow$ shrinkage) and rescue (depolymerization $\rightarrow$ growth). Several MAPs regulate thereby the shift between these two transitions. Microtubule minus (-) and plus ends (+) are indicated.

\section{Evolution of TACC adaptor proteins - conserved mitotic role and implications for nonmitotic functions}

TACC1 was the first human TACC protein family member that was identified and mapped close to the fibroblast growth factor receptor 1 (FGFR1) region at chromosome 8p11 (Still et al., 1999b). Similarly, TACC2 and TACC3 were identified and mapped to be physically linked to the corresponding FGFR2 and FGFR3 chromosomal regions 10q26 and 4p16, respectively (Still et al., 1999a, 2004). During evolution, two gene duplication processes gave rise to three distinct TACC/FGFR gene pairs (Still et al., 2004). Thus, three TACC isoforms have evolved in all vertebrates analyzed so far, except Xenopus sp., where only two isoforms, TACC3/Maskin (Xenopus laevis) and TACC2 (Xenopus tropicalis), have been reported (Klein et al., 2002; Still et al., 2004; O’Brien et al., 2005; Peset 
et al., 2005). In contrast, in organisms other than metazoans, only one single TACC isoform has been identified and characterized, i.e., Spc72 (Saccaromyces cerevisiae), Alp7 (Schizosaccaromyces pombe), TACC (Dictyostelium discoideum), TAC1 (Caenohabditis elegans), and DTACC (Drosophila melanogaster) (Chen et al., 1998; Gergely et al., 2000b; Sato et al., 2003, 2004; Srayko et al., 2003; Peset and Vernos, 2008; Samereier et al., 2011). TACC proteins are characterized by their highly conserved C-terminal coiled coil signature domain (TACC domain; Figure 2) (Gergely et al., 2000a; Still et al., 2004; Peset and Vernos, 2008; Gomez-Baldo et al., 2010; Ha et al., 2013b). However, looking at the evolutionary tree of coiled coil structure-containing proteins, the TACC family members form a subfamily, which is different from other typical coiled coil-rich proteins, like keratins, tropomyosins, and kinesins (Still et al., 2004; Gomez-Baldo et al., 2010).

The function of TACC orthologs in centrosomally controlled mitotic spindle assembly and dynamics remained conserved throughout evolution (Gergely, 2002; Still et al., 2004; Peset and Vernos, 2008). Interestingly, in addition to this conserved role, TACC family members of different species have obviously gained additional, mostly nuclear, functions during evolution (Still et al., 2004; Peset and Vernos, 2008), which are far less understood. For example, interaction of the zink finger protein friend of GATA1 (FOG1) or aryl hydrocarbon receptor nuclear translocator (ARNT) with murine TACC3 implicates a function of TACC3 as transcriptional coactivator (Table 1) (Sadek et al., 2000; Garriga-Canut and Orkin, 2004; Partch et al., 2011). In this line, murine TACC3 has been originally identified in a yeast two-hybrid screen for signal transducer and activator of transcription 5 (Stat5)-binding proteins; however, subsequent in-depth structural and functional interaction analysis in cell culture failed to confirm this interaction (Piekorz et al., 2002). Moreover, binding of the small nuclear ribonucleoproteins small nuclear ribonucleoprotein polypeptide $\mathrm{G}(\mathrm{SmG})$ and U6 snRNA-associated Sm-like protein LSm7 (LSM7) to TACC1 suggested a putative role of TACC1 in mRNA processing and homeostasis (Conte et al., 2002, 2003). A complex of TACC3 with tuberous sclerosis 2 (TSC2) has been implicated in the control of cytokinesis and maintenance of the nuclear envelope structure (Gomez-Baldo et al., 2010). Lastly, interactions of the different TACC family members with chromatin modifiers and transcriptional regulators, including glioma-amplified sequence 41 (GAS41), the nuclear receptors thyroid hormone receptor (TR) and retinoid acid receptor (RAR), the SWI/SNF chromatin remodeling complex, the translation initiation factor elF4E, and the histone acetyltransferase pCAF corroborate the putative and diverse functional portfolio that TACC proteins gained during evolution (overview in Table 1) (Still et al., 2004). Nevertheless, in vivo, the majority of these nonmitotic (mostly nuclear) functions of TACC proteins need to be critically subjected to an in-depth structure-function analysis in vivo, in particular, considering (i) that several TACC-binding partners have been originally identified by employing yeast two-hybrid screening using or fishing out the (rather sticky) TACC domain, and (ii) taking into account that TACC isoforms show rather defined expression profiles in vivo (cell type; proliferation status, i.e.,

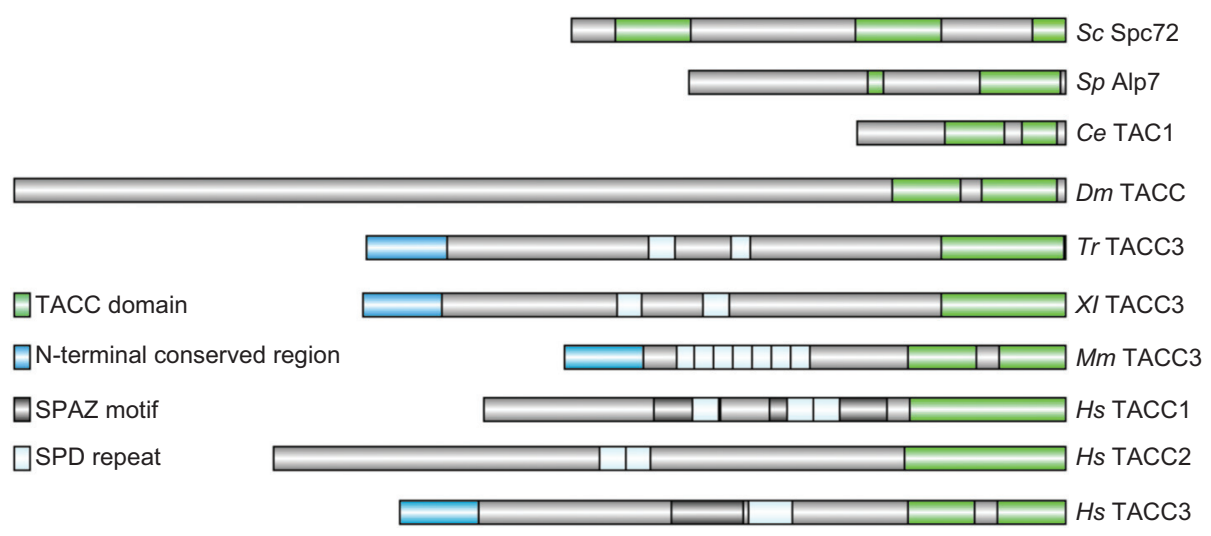

Figure 2 Domain organization of TACC protein family members.

Characteristic domains are indicated, including the highly conserved C-terminal TACC domain that is coiled coil-rich and present in all TACC proteins (green), the conserved first 100 amino acid residues at the N-terminus of TACC3 isoforms of higher eukaryotes (blue), random coil unstructured regions (gray), SPAZ motifs (dark gray), and the SPD-rich repeat region (blue). Species: Sc, Saccharomyces cereviceae; Sp, S. pombe; Tr, Takifugu rubripes; Xl, X. laevis; Hs, H. sapiens; Ms, M. musculus; Dm, D. melanogaster; Ce, C. elegans. Taken from Thakur (2012) and adapted after Peset and Vernos (2008). 


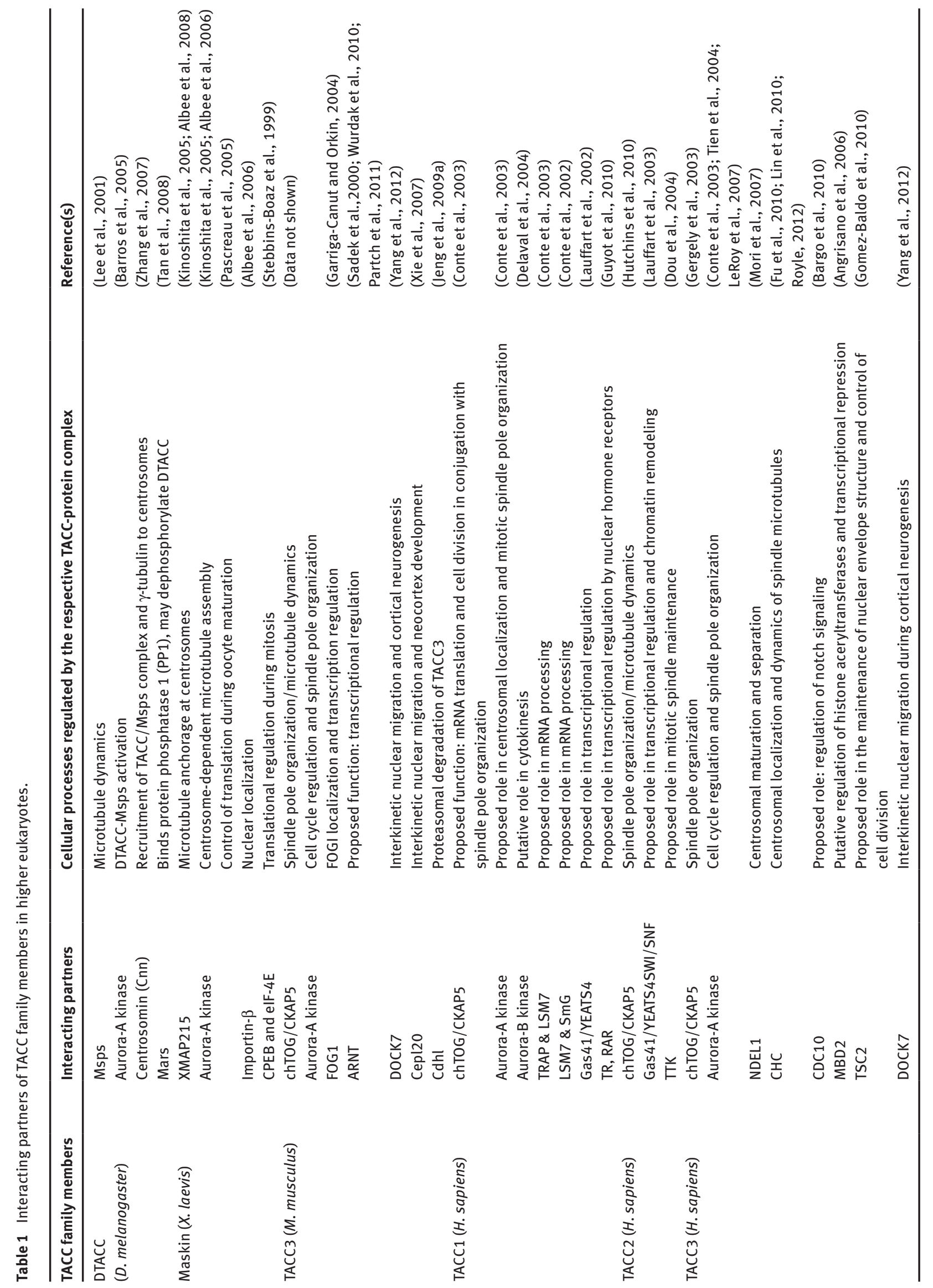


cycling vs. postmitotic), which do not always support the TACC-binding partner interactions observed under in vitro conditions.

\section{Insight into structural organization of TACC adaptor proteins}

All the TACC protein family members are characterized by their highly conserved C-terminal TACC signature domain comprised of approximately 200 amino acids, whereas the $\mathrm{N}$-terminus is rather variable in both sequence homology and length (Figure 2) (Gergely, 2002; Peset and Vernos, 2008; Ha et al., 2013a). TAC1, the TACC homolog in $C$. elegans, is the smallest member and consists only of the TACC domain without a variable N-terminus (Srayko et al., 2003). Spc72 from S. cerevisiae displays three separated coiled coil regions, one each at the $\mathrm{N}$ - and $\mathrm{C}$-terminus, and the third in the central part (Chen et al., 1998). Alp7, the TACC homolog in $S$. pombe, shows a smaller N-terminal variable region (Sato et al., 2004). All the other TACC family members contain longer $\mathrm{N}$-terminal variable regions adjacent to the TACC signature domain (Gergely, 2002; Peset and Vernos, 2008). As is obvious from the TACC3 interactome discussed below (Figure 3), the TACC domain is involved in the interaction with several known binding proteins, in particular, the microtubule polymerase chTOG/CKAP5.

The longer variable N-terminus of DTACC and also, in particular, of the vertebrate TACC3 isoforms contains binding sites for the mitotic serine-threonine kinase Aurora-A that phosphorylates three conserved serine residues (S863 in D-TACC; S33, S620, and S626 in X. laevis TACC3/Maskin; S34, S552, and S558 in Homo sapiens TACC3; and, putatively, S34, S341 and S347 in Mus musculus TACC3) (Giet et al., 2002; Barros et al., 2005; Kinoshita et al., 2005; Peset et al., 2005; LeRoy et al., 2007). As a consequence, Aurora-A phosphorylation of one particular serine (i.e., pS558 in human TACC3 and pS626 in TACC3/Maskin) determines the recruitment and differential subcellular localization of DTACC and TACC3 to centrosomes (pDTACC, pTACC3) compared to the localization along spindle microtubules toward their plus ends (DTACC, TACC3). Furthermore, the longer $\mathrm{N}$-terminus of the TACC family members contains
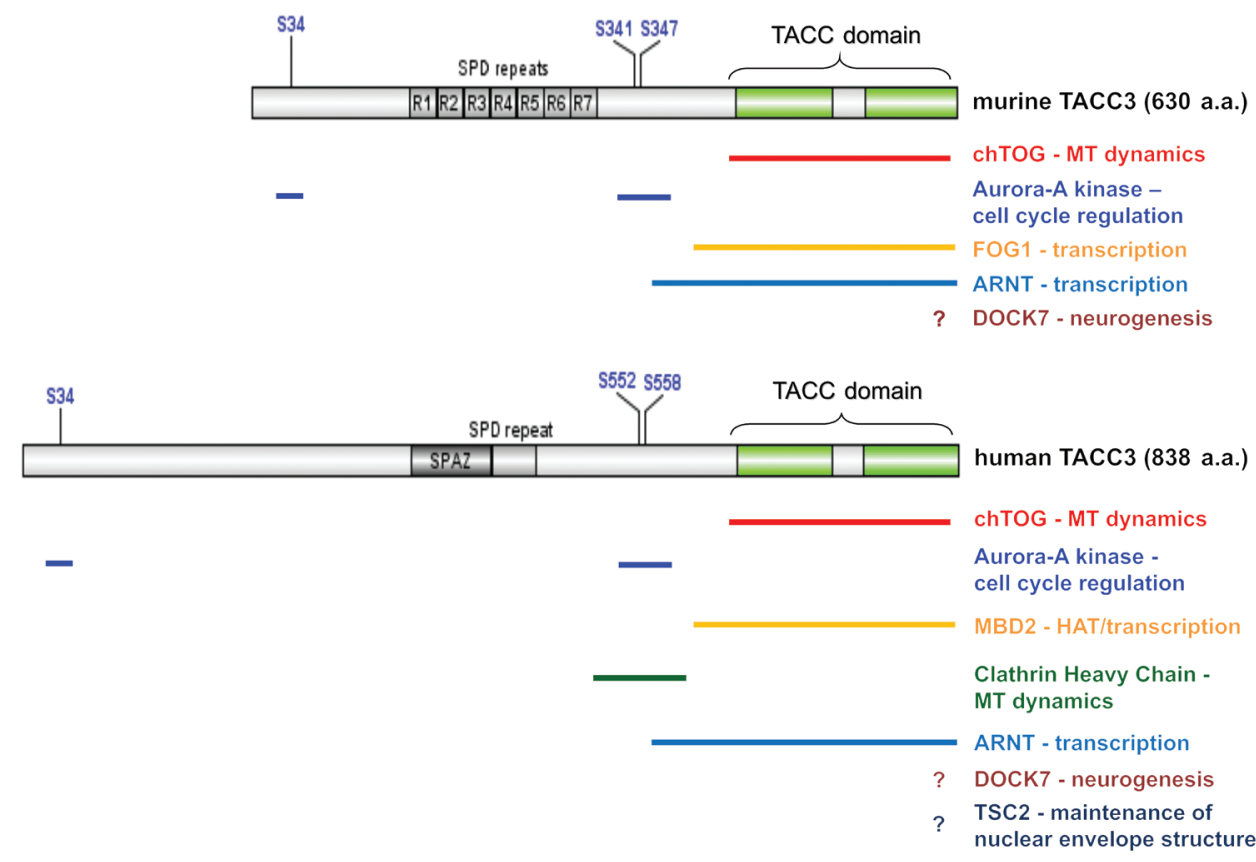

Figure 3 Insight into the TACC3 interactome.

Depicted are proteins known or proposed to interact with different domains of murine (upper part) and human (lower part) TACC3. The majority of the partner proteins depicted bind to the C-terminal coiled coil domain (TACC domain; green) of TACC3. As summarized in Table 1, besides ChTOG, interaction of TACC3 with several other proteins, which are involved in different cellular processes, has been described. The binding region(s) between DOCK7 (dedicator of cytokinesis 7) or TSC2 and TACC3 are currently unclear. The three conserved Aurora-A phosphorylation sites present in vertebrate TACC 3 isoforms are indicated. MT, microtubules; HAT, histone acetyltransferase. Taken from Thakur (2012). 
also a serine-proline (SP)-rich repeat region, named serine, prolin, and aspartate/glutamate (SPD/E)-rich repeats, and the so-called serine-prolin-Azu-1 (SPAZ) motifs (Figure 2) (Peset and Vernos, 2008). In murine TACC3, this region is characterized by the presence of seven perfect repeats of 24 amino acids each (Still et al., 2004). Interestingly, each repeat contains one PXXP motif, which represents a bona fide docking site for src homology 3 (SH3) domain-containing proteins (Kay et al., 2000). The role of protein-protein interaction via $\mathrm{SH} 3$ domains has been demonstrated in several proteins involved in cellular signal transduction. However, a putative function of the central repeat motifs present in TACC3 isoforms in protein-protein interaction remains to be established. Irrespective of this, the $\mathrm{N}$-termini of several TACC family members mediate the association with several proteins, including LSM7 and SmG (TACC1), eIF-4E and importin- $\beta$ (Maskin), Gas41 (TACC1 and 2), and TRAP (TACC1), which are all linked to rather diverse cellular processes as summarized in Table 1.
Centrosomes and the PCM are highly enriched with coiled coil-containing proteins (Andersen et al., 2003). Coiled coil motifs or domains are mainly dimeric to heptameric with a parallel or antiparallel orientation of the helices (Mikolajka et al., 2006). To analyze the oligomeric status and structural orientation of the conserved TACC domain, we purified murine TACC3 after bacterial overexpression and subjected it to biophysical characterization. Employing multi-angle light scattering (MALS) and analytical ultracentrifugation-sedimentation velocity (AUCSV) measurements, we observed that TACC3 displays a polydisperse, dimeric to oligomeric structure and a highly extended shape (Thakur, 2012). Moreover, negative staining and electron microscopy confirmed an elongated fiber-like structure of purified TACC3 with variable lengths (Figure 4). These findings are consistent with overexpression studies in eukaryotic cells where TACC proteins or the coiled coil-rich TACC domain per se formed highly ordered polymers with the capability to interact with microtubules (Gergely et al., 2000b).
A

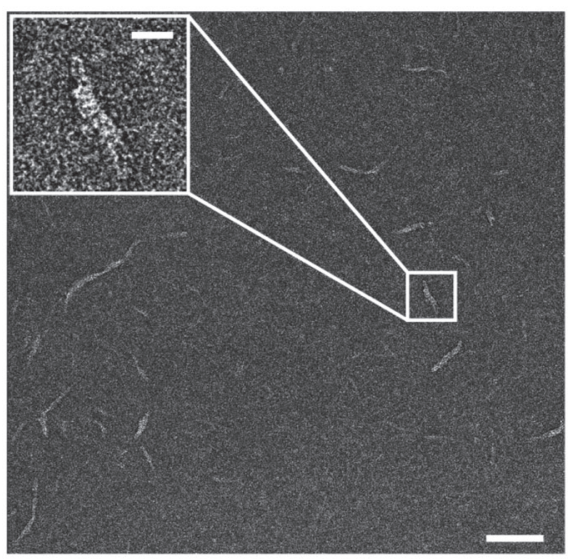

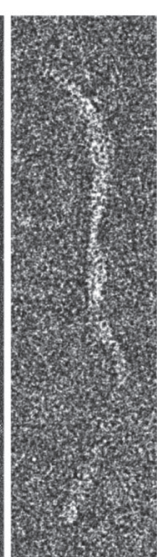

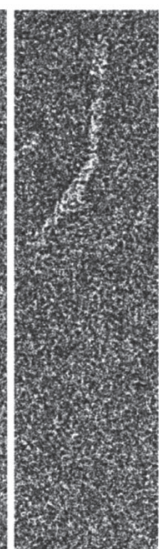

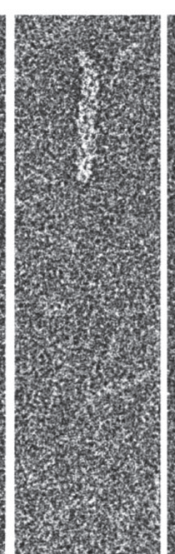

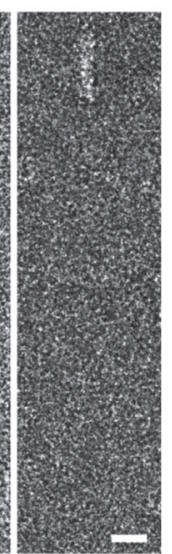

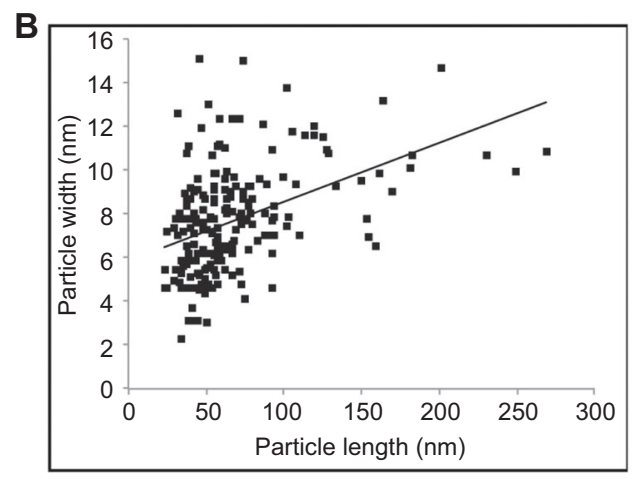

Figure 4 Electron microscopic analysis depicting an elongated structure and fiber-like appearance of highly purified murine TACC3 (A). Bar size in the left panel: $100 \mathrm{~nm}$. Enlarged single TACC3 fibers are shown in the right panels (bar: $10 \mathrm{~nm}$ ). (B) Quantitative analysis of fiberlike TACC3 molecules reveals average length and width variations of mostly $25-100 \mathrm{~nm}$ and 5-10 nm, respectively. For electron microscopic imaging, a Joel JEM-1400 electron microscope equipped with a LaB filament was used and operated at an acceleration voltage of $120 \mathrm{kV}$. Digital micrographs were taken at a corrected magnification of $53.800 \times$ and a defocus value of $-1.5 \mu \mathrm{m}$ using a $4 \mathrm{k} \times 4 \mathrm{k}$ CMOS camera F416 (TVIPS) at low-dose conditions. Taken from Thakur (2012). 


\section{TACC3 - interactome analysis and role in mitotic spindle function}

Insight into TACC3 function in microtubule dynamics and cell division has been recently elaborated by global interactome studies (Hubner et al., 2010; Hutchins et al., 2010; Neumann et al., 2010). One major effector protein binding to TACC3 via its TACC domain (Figure 3) represents the microtubule polymerase XMAP215/chTOG (Brouhard et al., 2008; Widlund et al., 2011) that colocalizes with TACC3 at the mitotic spindle apparatus (Figure 5A). The interaction of the TACC family members with the respective XMAP215 family member is evolutionary conserved and required for spindle pole organization and, in particular, microtubule dynamics during cell division (Bellanger and Gonczy, 2003; Gergely et al., 2003; Sato et al., 2004; Peset and Vernos, 2008). Deletion mapping of XMAP215 in $X$. laevis suggested that the $\mathrm{N}$-terminal part exhibits microtubule-stabilizing activity that stimulates microtubule growth at the plus ends (Popov et al., 2001; Kinoshita et al., 2002). In particular, the N-terminus of XMAP215/ chTOG proteins contains five evolutionary conserved TOG domains, which bind $\alpha, \beta$-tubulin heterodimers and, thereby, help to polymerize microtubules (Brouhard et al., 2008; Al-Bassam and Chang, 2011). Conversely, in oocyte extracts from $X$. laevis, it was demonstrated that the C-terminal part of XMAP215 was able to suppress the growth of microtubules by promoting microtubule catastrophes (Popov et al., 2001). Formation of the TACC3/ Maskin-XMAP215/chTOG complex appears to antagonize the microtubule depolymerase activity of the kinesin-like protein XKCM1/MCAK at the plus poles (Kinoshita et al., 2005) and, independent from XKCM1/MCAK function, to stimulate assembly of microtubules at centrosomes (Barr and Gergely, 2008). Taken these findings into account, it seems that TACC3/Maskin functions as an adaptor protein (Hood and Royle, 2011), where binding of the TACC domain to XMAP215/chTOG 'engages' the C-terminal part of XMAP215/chTOG and, thereby, inhibits its microtubule catastrophe-promoting activity. Therefore, during mitotic cell division, the TACC3/Maskin-XMAP215/chTOG protein complex likely causes a shift of the dynamic equilibrium toward the microtubule rescue status (Figure 1). Upon metaphase-anaphase transition and mitotic exit, the TACC3 levels strongly decline by Cdh1-dependent ubiquitination and proteasomal degradation (Jeng et al., 2009a). As a consequence, a gradual increase in depolymerization activity by the 'TACC-free' $\mathrm{C}$-terminal domain of XMAP215/chTOG may then lead to a dynamic equilibrium
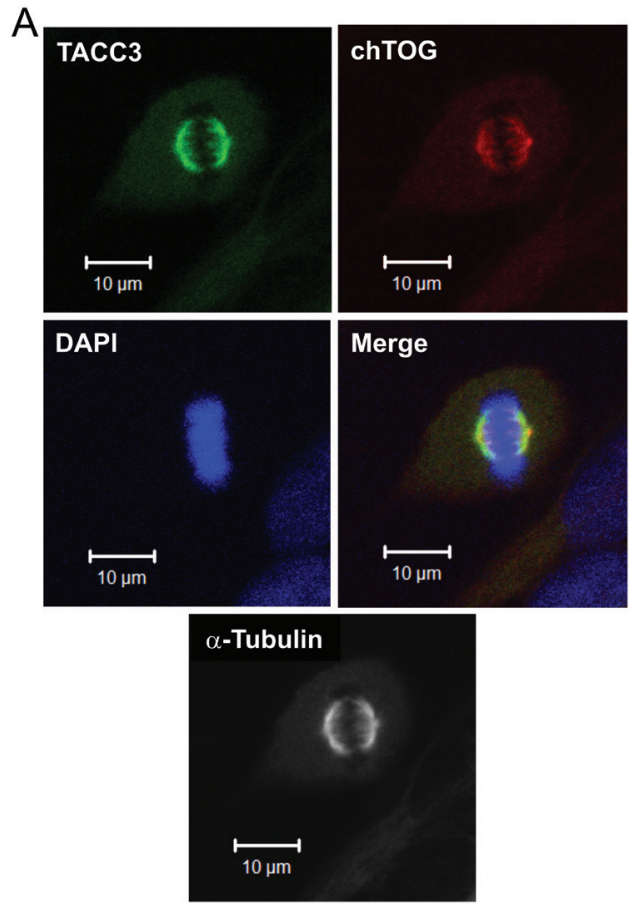
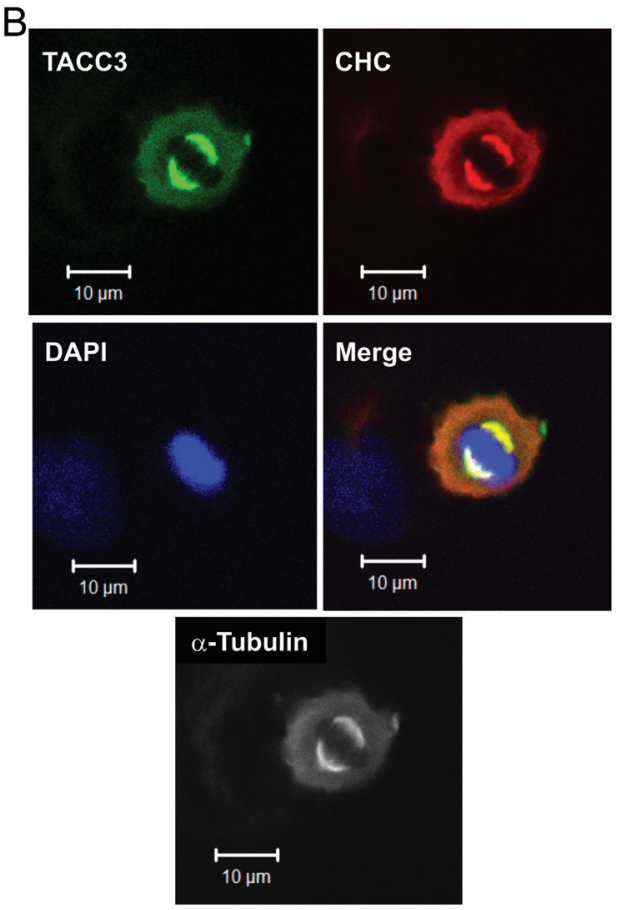

Figure 5 Subcellular colocalization of TACC 3 with chTOG and CHC (clathrin heavy chain) in mitotic Hela cells. Images of metaphases were collected under a Zeiss CLSM510-Meta microscope at a $63 \times$ magnification.

(A) At centrosomes and the spindle apparatus, TACC3 colocalizes with chTOG and microtubules. (B) CHC colocalizes with TACC3 at centrosomes and a diffused region around the spindle poles. DAPI was used to detect DNA. Taken from Thakur (2012). 

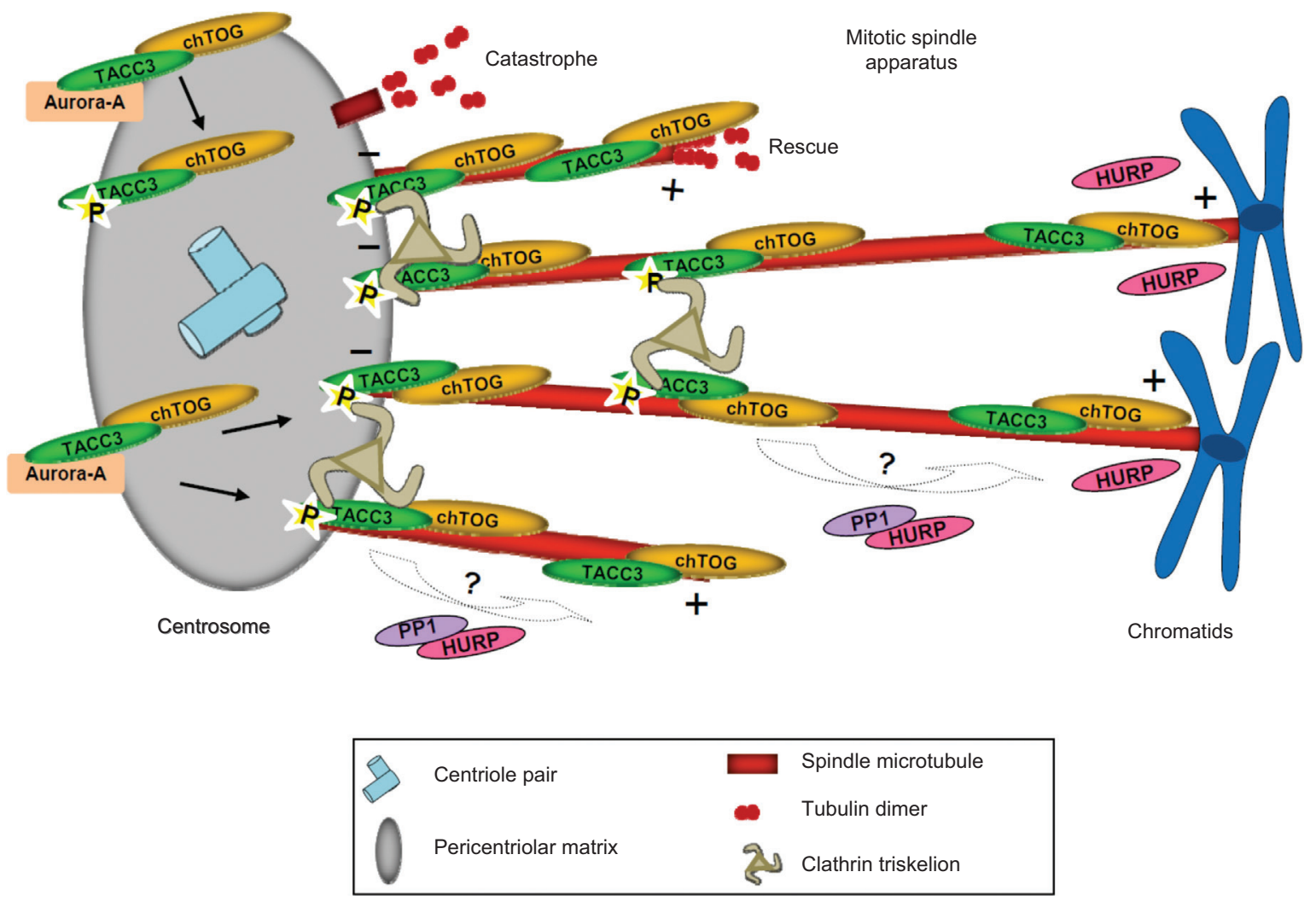

Figure 6 Schematic model summarizing the role of TACC3 in the regulation of microtubule dynamics at the mitotic spindle apparatus. TACC 3 in a complex with chTOG localizes at the centrosome and along spindle microtubules in an Aurora-A kinase-regulated manner. The TACC3 (pS558)-chTOG complex interacts with the clathrin triskelion, thereby, mediating microtubule growth kinetics and stability both at the longitudinal level and by providing microtubule cross linking (Booth et al., 2011; Royle, 2012). Interestingly, the RanGTP and Aurora-A regulated and K-fiber-localized HURP may recruit protein phosphatase 1 (PP1) to the mitotic spindle apparatus. It is, therefore, tempting to speculate that TACC3 (pS558) undergoes dephosphorylation by the HURP-controlled PP1 complex prior to localization toward the microtubule plus poles as suggested for DTACC (Tan et al., 2008). Model adapted and taken from Thakur (2012).

shift toward the microtubule catastrophe status. Of note, this proposed mechanism may be evolutionary conserved given the very high sequence identity of the C-termini of mammalian chTOG proteins when, e.g., compared to the C-terminus of XMAP215 from X. laevis (Thakur, 2012). Moreover, the 'KKIGSK' sequence and phosphorylation motif present in the C-terminus of XMAP215, which plays a role in (possibly TACC3/Maskin-dependent) centrosomal targeting of XMAP215 (Popov et al., 2001), is also completely conserved in mammalian chTOG proteins. Taken together, although an in-depth structural TACC3chTOG interaction mapping has not been described yet, a model can be envisioned where TACC3/Maskin regulates spindle microtubule dynamics during mitotic progression through the control of the spatiotemporal localization of XMAP215/chTOG and its microtubule (de)polymerizing activity (Figure 6).

Interestingly, clathrin heavy chain (CHC), which has been recently identified as the interacting partner for the
TACC3-chTOG protein complex (Fu et al., 2010; Hubner et al., 2010; Lin et al., 2010; Booth et al., 2011), plays an essential role in the organization of the mitotic spindle where CHC perfectly colocalizes with TACC3 (Figure 5B). Binding of $\mathrm{CHC}$ to TACC3/Maskin occurs, in contrast to chTOG, outside of the TACC domain and requires AuroraA-mediated phosphorylation at serine residues 620 and 626 (Maskin) or 558 (human TACC3; Figure 3) (Fu et al., 2010; Lin et al., 2010). In the following clathrin heavy chain 17 (CHC17), but not the related isoform CHC22, was shown to control spindle pole integrity during early mitosis by stabilizing centrosomal chTOG (Foraker et al., 2012). Moreover, elegant work by Booth et al. demonstrated that the TACC3-chTOG-CHC protein complex is crucial for the stabilization of intermicrotubule bridges and kinetochore fibers, the latter mediating chromosomal movement during mitosis (Booth et al., 2011). Rapid inactivation of the TACC3-chTOG-clathrin axis at defined mitotic stages proved that clathrin, which is recruited to 
microtubules by phosphorylated TACC3 (Fu et al., 2011; Royle, 2012), is essential for both spindle assembly and subsequent spindle function (Cheeseman et al., 2013). In summary, TACC3 functions as an adaptor protein and a recruitment factor for both chTOG and clathrin to ensure proper mitotic spindle dynamics and function.

\section{TACC3 - expression pattern and biological role}

Mammalian TACC isoforms display differences in the temporal and spatial expression profile as well as in the subcellular localization pattern (Gergely et al., 2000a; Piekorz et al., 2002; Schuendeln et al., 2004). Human TACC3 was initially identified as gene overexpressed in multiple myeloma (Still et al., 1999a; Eslinger et al., 2010) and various other cancer cell lines and tumor cell types (Ha et al., 2013b). TACC3 is expressed at high levels during embryogenesis and postnatally in proliferating and regenerative cell types, including the hematopoietic system, reproductive organs, and epithelial cells (Hao et al., 2002; Piekorz et al., 2002; Schuendeln et al., 2004). In contrast, TACC2 is found predominantly in postmitotic tissues like the brain and heart (Schuendeln et al., 2004), whereas TACC1 shows a more ubiquitous expression pattern in vivo (Lauffart et al., 2006). During cell cycle progression, TACC3 mRNA and protein levels increase dramatically in the late $\mathrm{S}$ - and $\mathrm{G}_{2}-/ \mathrm{M}$-phase (Piekorz et al., 2002), followed by Cdh1-regulated proteasomal degradation of TACC3 during mitotic exit (Jeng et al., 2009b).

Studies employing mouse knockout models revealed a vital role of TACC3 during embryonic development. Gene deficiency for TACC3 results in embryonic lethality associated with aneuploidy, activation of the mitotic spindle assembly, and postmitotic p53-p21 ${ }^{\mathrm{WAF}}$ checkpoints, apoptosis, impaired proliferation, and overall strong growth retardation (Piekorz et al., 2002; Yao et al., 2007). At this, TACC3 plays, e.g., an essential role in hematopoietic stem cell function or mesenchymal cell expansion that is required for proper formation of the axial skeleton (Yao et al., 2007). Comparable effects, including an induction of growth arrest (by triggering the cellular senescence program) or apoptosis, both dependent on the $\mathrm{p} 53 / \mathrm{G}_{1}$ checkpoint status, have been observed when TACC 3 was depleted by RNA interference in various cell culture models (Schmidt et al., 2010a,b; Schneider et al., 2007, 2008). In contrast, TACC2 is dispensable for normal development, and TACC2 gene deficiency did not cause any detectable mitotic or proliferative defects (Schuendeln et al., 2004). Moreover, double deficiency for TACC2 and TACC3 did not aggravate the phenotype of TACC3-deficient embryos. As a TACC1 deficiency has not been described yet, possible redundant roles between TACC1 and TACC 2 cannot be assessed. Taken together, the reason(s) why mammalians evolved three TACC isoforms with possibly specific roles as well as the degree of functional redundancy between these isoforms remain(s) unclear.

\section{The emerging pathobiological role of TACC proteins in human cancer}

TACC genes have been originally discovered in genomic regions that are amplified in breast tumors and multiple myeloma (Still et al., 1999b). Mutations or an altered expression of TACC1 and TACC3 have been subsequently linked to the etiology of breast, ovarian, bladder, nonsmall-cell lung cancer, prostate, and melanoma tumors (Cully et al., 2005; Lauffart et al., 2005; Jung et al., 2006; Kiemeney et al., 2010; Hodis et al., 2012; Ha et al., 2013a). A role in the androgen-mediated growth of prostate cancer has been suggested for TACC2 (Takayama et al., 2012). Moreover, the TACC3 gene is amplified in glioma tumors at the genomic level. These tumors show a grade-specific upregulation of TACC3 expression, which is highest in grade IV glioma, a tumor type with poor prognosis (Duncan et al., 2010). Interestingly, intrachromosomal FGFR1-TACC1 and FGFR3-TACC3 gene fusions have also been recently detected in subsets of glioblastoma and bladder cancer patients (Singh et al., 2012; Williams et al., 2012; Parker et al., 2011). These fusion proteins display a constitutive FGFR tyrosine kinase activity presumably activating downstream signaling effector pathways, localize to mitotic spindle poles obviously due to the presence of the C-terminally fused TACC domain, are associated with aneuploidy, and display pro-proliferative and oncogenic activities both in vitro and in vivo. In this regard, TACC3 influences PI3K/Akt and ERK signaling associated with tumorigenic epithelial-mesenchymal transition (EMT) and cell migration/invasion (Ha et al., 2013b).

Moreover, TACC3 linked [e.g., hepatoma up-regulated protein (HURP)] or interacting (e.g., chTOG or TSC2) proteins can be as well altered at the level of structure or expression in different transformed tumor cell types (overview in Ha et al., 2013a), therefore, potentially contributing to tumorigenesis. For example, chTOG and HURP were identified as overexpressed genes in human hepatic carcinomas (Charrasse et al., 1995; Tsou et al., 2003), and mutations of the tumor suppressor protein 
TSC2 were initially observed in renal carcinogenesis and hepatic hemangiomatosis (Kobayashi et al., 1999).

Taking these findings into account, it is tempting to speculate that members of the mitotic Aurora-A-TACC3chTOG axis act as 'driver' factors in tumor development and, hence, represent potential therapeutic targets. Indeed, conditional loss of the TACC3 gene expression leads to apoptosis and tumor regression in vivo in the $\mathrm{p} 53^{\%}$ mouse sarcolymphoma model (Yao et al., 2011). A role of TACC3 in chemoresistance and the cellular response to antimitotics can also be envisioned, given that RNAimediated TACC3 depletion or treatment with the TACC3 inhibitor KSH101 (Wurdak et al., 2010) sensitizes tumor cells to the antiproliferative and apoptotic effects of paclitaxel (Schneider et al., 2008; Schmidt et al., 2010a; Cappell et al., 2012). Consistent with this, TACC depletion renders cells from $D$. discoideum also to be hypersensitive against the microtubule-depolymerizing drug thiabendazole (Samereier et al., 2011).

\section{Conclusion and open questions}

The Aurora-A kinase regulated TACC3-chTOG-CHC protein complex plays a pivotal role in centrosomal function and mitotic spindle assembly and dynamics (Figure 6). Despite the current insight into the TACC isoform expression and function (Peset and Vernos, 2008; Zyss and Gergely, 2009; Royle, 2012; Ha et al., 2013b), various molecular and cellular aspects of TACC (patho)biology await further investigation. For instance, there is lack of insight into the three-dimensional structure of the TACC3 domains mediating the interaction with chTOG or clathrin heavy chain. Another issue is the analysis of the role of dephosphorylation as regulatory mechanism to

\section{References}

Al-Bassam, J. and Chang, F. (2011). Regulation of microtubule dynamics by TOG-domain proteins XMAP215/Dis1 and CLASP. Trends Cell Biol. 21, 604-614.

Andersen, J.S., Wilkinson, C.J., Mayor, T., Mortensen, P., Nigg, E.A., and Mann, M. (2003). Proteomic characterization of the human centrosome by protein correlation profiling. Nature 426 , 570-574.

Azimzadeh, J. and Bornens, M. (2005). The centrosome in evolution. In Centrosomes in Development and Disease (Weinheim, Germany: Wiley-VCH Verlag GmbH \& Co. KGaA), pp. 93-122.

Barr, A.R. and Gergely, F. (2008). MCAK-independent functions of ch-Tog/XMAP215 in microtubule plus-end dynamics. Mol. Cell Biol. 28, 7199-7211. control the mitotic pools of pTACC3 vs. TACC3. An interaction of DTACC with the microtubule-associated protein Mars, which binds directly to protein phosphatase PP1, thereby promoting DTACC dephosphorylation, has been reported (Tan et al., 2008). Interestingly, HURP represents the Mars homolog in higher vertebrates that localizes to kinetochore microtubules ( $\mathrm{k}$-fibers) close to chromosomes (Sillje et al., 2006). HURP was described as an oncogenic target of Aurora-A kinase (Yu et al., 2005), thereby, controlling the microtubule-binding capacity of HURP (Wong et al., 2008). Thus, HURP may recruit PP1 or a currently unknown phosphatase to the microtubules and, hence, control spindle dynamics by balancing pTACC3/TACC3 levels (Figure 6). Lastly, structure-function analyses of putative 'driver' TACC1 and TACC3 mutations in human cancers with respect to mitotic loss-of-function or gain-offunction phenotypes is required. The latter apply, in particular, to genomic instability, checkpoint activation, and oncogenic signaling.

Acknowledgments: We thank our colleagues Astrid Hoeppner, Cordula Kruse, Klaus Schulze-Osthoff, Reiner Jänicke, Jürgen Scheller, and all former and current members of the Institute for Biochemistry and Molecular Biology II for support and discussions. We gratefully acknowledge financial support by a Ph.D. fellowship of the NRW graduate school 'BioStruct - Biological Structures in Molecular Medicine and Biotechnology' (to H.C.T.), the DFG (SFB 728 to R.P.P.), and the research commission of the medical faculty of the Heinrich-Heine-University (to R.P.P. and M.R.A). E.K.F. and M.R.A. thank the BMBF (NGFNplus program, grant 01GS08100) for their financial support.

Received May 16, 2013; accepted June 18, 2013; previously published online June 20, 2013
Barros, T.P., Kinoshita, K., Hyman, A.A., and Raff, J.W. (2005). Aurora A activates D-TACC-Msps complexes exclusively at centrosomes to stabilize centrosomal microtubules. J. Cell Biol. 170, 1039-1046.

Bellanger, J.M. and Gonczy, P. (2003). TAC-1 and ZYG-9 form a complex that promotes microtubule assembly in $C$. elegans embryos. Curr. Biol. 13, 1488-1498.

Booth, D.G., Hood, F.E., Prior, I.A., and Royle, S.J. (2011). A TACC3/ch-TOG/clathrin complex stabilises kinetochore fibres by inter-microtubule bridging. EMBO J. 30, 906-919.

Bornens, M. (2002). Centrosome composition and microtubule anchoring mechanisms. Curr. Opin. Cell Biol. 14, 25-34. 
Bornens, M. (2012). The centrosome in cells and organisms. Science 335, 422-426.

Brouhard, G.J., Stear, J.H., Noetzel, T.L., Al-Bassam, J., Kinoshita, K., Harrison, S.C., Howard, J., and Hyman, A.A. (2008). XMAP215 is a processive microtubule polymerase. Cell 132, 79-88.

Cappell, K.M., Sinnott, R., Taus, P., Maxfield, K., Scarbrough, M., and Whitehurst, A.W. (2012). Multiple cancer testis antigens function to support tumor cell mitotic fidelity. Mol. Cell Biol. 32, 4131-4140.

Charrasse, S., Mazel, M., Taviaux, S., Berta, P., Chow, T., and Larroque, C. (1995). Characterization of the cDNA and pattern of expression of a new gene over-expressed in human hepatomas and colonic tumors. Eur. J. Biochem. 234, 406-413.

Cheeseman, L.P., Harry, E.F., McAinsh, A.D., Prior, I.A., and Royle, S.J. (2013). Specific removal of TACC3/ch-TOG/clathrin at metaphase deregulates kinetochore fiber tension. J. Cell Sci. 126, 2102-2113.

Chen, X.P., Yin, H., and Huffaker, T.C. (1998). The yeast spindle pole body component $S p c 72 p$ interacts with Stu2p and is required for proper microtubule assembly. J. Cell Biol. 141, 1169-1179.

Compton, D.A. (2000). Spindle assembly in animal cells. Annu. Rev. Biochem. 69, 95-114.

Conte, N., Charafe-Jauffret, E., Delaval, B., Adelaide, J., Ginestier, C., Geneix, J., Isnardon, D., Jacquemier, J., and Birnbaum, D. (2002). Carcinogenesis and translational controls: TACC1 is down-regulated in human cancers and associates with mRNA regulators. Oncogene 21, 5619-5630.

Conte, N., Delaval, B., Ginestier, C., Ferrand, A., Isnardon, D., Larroque, C., Prigent, C., Seraphin, B., Jacquemier, J., and Birnbaum, D. (2003). TACC1-chTOG-Aurora A protein complex in breast cancer. Oncogene 22, 8102-8116.

Cully, M., Shiu, J., Piekorz, R.P., Muller, W.J., Done, S.J., and Mak, T.W. (2005). Transforming acidic coiled coil 1 promotes transformation and mammary tumorigenesis. Cancer Res. 65, 10363-10370.

DeLuca, J.G. and Musacchio, A. (2012). Structural organization of the kinetochore-microtubule interface. Curr. Opin. Cell Biol. 24, 48-56.

Desai, A. and Mitchison, T.J. (1997). Microtubule polymerization dynamics. Annu. Rev. Cell Dev. Biol. 13, 83-117.

Duncan, C.G., Killela, P.J., Payne, C.A., Lampson, B., Chen, W.C., Liu, J., Solomon, D., Waldman, T., Towers, A.J., Gregory, S.G., et al. (2010). Integrated genomic analyses identify ERRFl1 and TACC3 as glioblastoma-targeted genes. Oncotarget 1, 265-277.

Eslinger, M.R., Lauffart, B., and Still, I.H. (2010). TACC3 (transforming, acidic coiled-coil containing protein 3). Atlas Genet. Cytogenet. Oncol. Haematol. 14, 527-553

Foraker, A.B., Camus, S.M., Evans, T.M., Majeed, S.R., Chen, C.Y., Taner, S.B., Correa, I.R., Jr., Doxsey, S.J., and Brodsky, F.M. (2012). Clathrin promotes centrosome integrity in early mitosis through stabilization of centrosomal ch-TOG. J. Cell Biol. 198, 591-605.

Fu, W., Jiang, Q., and Zhang, C. (2011). Novel functions of endocytic player clathrin in mitosis. Cell Res. 21, 1655-1661.

Fu, W., Tao, W., Zheng, P., Fu, J., Bian, M., Jiang, Q., Clarke, P.R., and Zhang, C. (2010). Clathrin recruits phosphorylated TACC3 to spindle poles for bipolar spindle assembly and chromosome alignment. J. Cell Sci. 123, 3645-3651.

Gardner, M.K., Zanic, M., and Howard, J. (2013). Microtubule catastrophe and rescue. Curr. Opin. Cell Biol. 25, 14-22.
Garriga-Canut, M. and Orkin, S.H. (2004). Transforming acidic coiled-coil protein 3 (TACC3) controls friend of GATA-1 (FOG-1) subcellular localization and regulates the association between GATA-1 and FOG-1 during hematopoiesis. J. Biol. Chem. 279, 23597-23605.

Gergely, F. (2002). Centrosomal TACCtics. Bioessays 24, 915-925.

Gergely, F., Draviam, V.M., and Raff, J.W. (2003). The ch-TOG/ XMAP215 protein is essential for spindle pole organization in human somatic cells. Genes Dev. 17, 336-341.

Gergely, F., Karlsson, C., Still, I., Cowell, J., Kilmartin, J., and Raff, J.W. (2000a). The TACC domain identifies a family of centrosomal proteins that can interact with microtubules. Proc. Natl. Acad. Sci. USA 97, 14352-14357.

Gergely, F., Kidd, D., Jeffers, K., Wakefield, J.G., and Raff, J.W. (2000b). D-TACC: a novel centrosomal protein required for normal spindle function in the early Drosophila embryo. EMBO J. 19, 241-252.

Giet, R., McLean, D., Descamps, S., Lee, M.J., Raff, J.W., Prigent, C., and Glover, D.M. (2002). Drosophila Aurora A kinase is required to localize D-TACC to centrosomes and to regulate astral microtubules. J. Cell Biol. 156, 437-451.

Gomez-Baldo, L., Schmidt, S., Maxwell, C.A., Bonifaci, N., Gabaldon, T., Vidalain, P.O., Senapedis, W., Kletke, A., Rosing, M., Barnekow, A., et al. (2010). TACC3-TSC2 maintains nuclear envelope structure and controls cell division. Cell Cycle 9, 1143-1155.

Ha, G.H., Kim, J.L., and Breuer, E.K. (2013a). Transforming acidic coiled-coil proteins (TACCS) in human cancer. Cancer Lett. DOI: 10.1016/j.canlet.2013.04.022. Epub April 23, 2013.

Ha, G.H., Park, J.S., and Breuer, E.K. (2013b). TACC3 promotes epithelial-mesenchymal transition (EMT) through the activation of PI3K/Akt and ERK signaling pathways. Cancer Lett. 332, 63-73.

Hao, Z., Stoler, M.H., Sen, B., Shore, A., Westbrook, A., Flickinger, C.J., Herr, J.C., and Coonrod, S.A. (2002). TACC3 expression and localization in the murine egg and ovary. Mol. Reprod. Dev. 63, 291-299.

Hodis, E., Watson, I.R., Kryukov, G.V., Arold, S.T., Imielinski, M., Theurillat, J.P., Nickerson, E., Auclair, D., Li, L., Place, C., et al. (2012). A landscape of driver mutations in melanoma. Cell 150, 251-263.

Hood, F.E. and Royle, S.J. (2011). Pulling it together: the mitotic function of TACC3. BioArchitecture 1, 105-109.

Hubner, N.C., Bird, A.W., Cox, J., Splettstoesser, B., Bandilla, P., Poser, I., Hyman, A., and Mann, M. (2010). Quantitative proteomics combined with BAC TransgeneOmics reveals in vivo protein interactions. J. Cell Biol. 189, 739-754.

Hutchins, J.R., Toyoda, Y., Hegemann, B., Poser, I., Heriche, J.K., Sykora, M.M., Augsburg, M., Hudecz, O., Buschhorn, B.A., Bulkescher, J., et al. (2010). Systematic analysis of human protein complexes identifies chromosome segregation proteins. Science 328, 593-599.

Jeng, J.-C., Lin, Y.-M., Lin, C.-H., and Shih, H.-M. (2009a). Cdh1 controls the stability of TACC3. Cell Cycle 8, 3537-3544.

Jeng, J.C., Lin, Y.M., Lin, C.H., and Shih, H.M. (2009b). Cdh1 controls the stability of TACC3. Cell Cycle 8, 3529-3536.

Jung, C.K., Jung, J.H., Park, G.S., Lee, A., Kang, C.S., and Lee, K.Y. (2006). Expression of transforming acidic coiled-coil containing protein 3 is a novel independent prognostic marker in non-small cell lung cancer. Pathol. Int. 56, 503-509. 
Kay, B.K., Williamson, M.P., and Sudol, M. (2000). The importance of being proline: the interaction of proline-rich motifs in signaling proteins with their cognate domains. FASEB J. 14, 231-241.

Khodjakov, A. and Rieder, C.L. (2009). The nature of cell-cycle checkpoints: facts and fallacies. J. Biol. 8, 88 .

Kiemeney, L.A., Sulem, P., Besenbacher, S., Vermeulen, S.H., Sigurdsson, A., Thorleifsson, G., Gudbjartsson, D.F., Stacey, S.N., Gudmundsson, J., Zanon, C., et al. (2010). A sequence variant at $4 \mathrm{p} 16.3$ confers susceptibility to urinary bladder cancer. Nat. Genet. 42, 415-419.

Kinoshita, K., Habermann, B., and Hyman, A.A. (2002). XMAP215: a key component of the dynamic microtubule cytoskeleton. Trends Cell Biol. 12, 267-273.

Kinoshita, K., Noetzel, T.L., Pelletier, L., Mechtler, K., Drechsel, D.N., Schwager, A., Lee, M., Raff, J.W., and Hyman, A.A. (2005). Aurora A phosphorylation of TACC3/maskin is required for centrosome-dependent microtubule assembly in mitosis. J. Cell Biol. 170, 1047-1055.

Klein, S.L., Strausberg, R.L., Wagner, L., Pontius, J., Clifton, S.W., and Richardson, P. (2002). Genetic and genomic tools for Xenopus research: the NIH Xenopus initiative. Dev. Dyn. 225, 384-391.

Kobayashi, T., Minowa, O., Kuno, J., Mitani, H., Hino, O., and Noda, T. (1999). Renal carcinogenesis, hepatic hemangiomatosis, and embryonic lethality caused by a germ-line Tsc 2 mutation in mice. Cancer Res. 59, 1206-1211.

Lauffart, B., Dimatteo, A., Vaughan, M.M., Cincotta, M.A., Black, J.D., and Still, I.H. (2006). Temporal and spatial expression of TACC1 in the mouse and human. Dev. Dyn. 235, 1638-1647.

Lauffart, B., Vaughan, M., Eddy, R., Chervinsky, D., DiCioccio, R., Black, J., and Still, I. (2005). Aberrations of TACC1 and TACC3 are associated with ovarian cancer. BMC Women's Health 5, 8.

LeRoy, P.J., Hunter, J.J., Hoar, K.M., Burke, K.E., Shinde, V., Ruan, J., Bowman, D., Galvin, K., and Ecsedy, J.A. (2007). Localization of human TACC3 to mitotic spindles is mediated by phosphorylation on Ser558 by Aurora A: a novel pharmacodynamic method for measuring Aurora A activity. Cancer Res. 67, 5362-5370.

Lin, C.H., Hu, C.K., and Shih, H.M. (2010). Clathrin heavy chain mediates TACC3 targeting to mitotic spindles to ensure spindle stability. J. Cell Biol. 189, 1097-1105.

Mikolajka, A., Yan, X., Popowicz, G.M., Smialowski, P., Nigg, E.A., and Holak, T.A. (2006). Structure of the N-terminal domain of the FOP (FGFR1OP) protein and implications for its dimerization and centrosomal localization. J. Mol. Biol. 359, 863-875.

Mitchison, T. and Kirschner, M. (1984). Dynamic instability of microtubule growth. Nature 312, 237-242.

Musacchio, A. (2011). Spindle assembly checkpoint: the third decade. Philos. Trans. R. Soc. Lond. B Biol. Sci. 366, 3595-3604.

Neumann, B., Walter, T., Heriche, J.K., Bulkescher, J., Erfle, H., Conrad, C., Rogers, P., Poser, I., Held, M., Liebel, U., et al. (2010). Phenotypic profiling of the human genome by time-lapse microscopy reveals cell division genes. Nature 464 , 721-727.

Nigg, E.A. and Raff, J.W. (2009). Centrioles, centrosomes, and cilia in health and disease. Cell 139, 663-678.

O’Brien, L.L., Albee, A.J., Liu, L., Tao, W., Dobrzyn, P., Lizarraga, S.B., and Wiese, C. (2005). The Xenopus TACC homologue, maskin, functions in mitotic spindle assembly. Mol. Biol. Cell $16,2836-2847$.
Parker, B.C., Annala, M.J., Cogdell, D.E., Granberg, K.J., Sun, Y., Ji, P., Li, X., Gumin, J., Zheng, H., Hu, L., et al. (2011). Coactivators necessary for transcriptional output of the hypoxia inducible factor, HIF, are directly recruited by ARNT PAS-B. Proc. Natl. Acad. Sci. USA 108, 7739-7744.

Peset, I., Seiler, J., Sardon, T., Bejarano, L.A., Rybina, S., and Vernos, I. (2005). Function and regulation of Maskin, a TACC family protein, in microtubule growth during mitosis. J. Cell Biol. 170, 1057-1066.

Peset, I. and Vernos, I. (2008). The TACC proteins: TACC-ling microtubule dynamics and centrosome function. Trends Cell Biol. 18, 379-388.

Piekorz, R.P., Hoffmeyer, A., Duntsch, C.D., McKay, C., Nakajima, H., Sexl, V., Snyder, L., Rehg, J., and Ihle, J.N. (2002). The centrosomal protein TACC3 is essential for hematopoietic stem cell function and genetically interfaces with p53-regulated apoptosis. EMBO J. 21, 653-664.

Popov, A.V., Pozniakovsky, A., Arnal, I., Antony, C., Ashford, A.J., Kinoshita, K., Tournebize, R., Hyman, A.A., and Karsenti, E. (2001). XMAP215 regulates microtubule dynamics through two distinct domains. EMBO J. 20, 397-410.

Raff, J.W. (2002). Centrosomes and cancer: lessons from a TACC. Trends Cell Biol. 12, 222-225.

Rieder, C.L. (2011). Mitosis in vertebrates: the G2/M and M/A transitions and their associated checkpoints. Chromosome Res. 19, 291-306.

Rieder, C.L. and Maiato, H. (2004). Stuck in division or passing through: what happens when cells cannot satisfy the spindle assembly checkpoint. Dev. Cell 7, 637-51.

Royle, S.J. (2012). The role of clathrin in mitotic spindle organisation. J. Cell Sci. 125, 19-28.

Sadek, C.M., Jalaguier, S., Feeney, E.P., Aitola, M., Damdimopoulos, A.E., Pelto-Huikko, M., and Gustafsson, J.A. (2000). Isolation and characterization of AINT: a novel ARNT interacting protein expressed during murine embryonic development. Mech. Dev. 97, 13-26.

Samereier, M., Baumann, O., Meyer, I., and Graf, R. (2011). Analysis of Dictyostelium TACC reveals differential interactions with CP224 and unusual dynamics of Dictyostelium microtubules. Cell. Mol. Life Sci. 68, 275-287.

Sato, M., Koonrugsa, N., Toda, T., Vardy, L., Tournier, S., and Millar, J.B. (2003). Deletion of Mia1/Alp7 activates Mad2-dependent spindle assembly checkpoint in fission yeast. Nat. Cell Biol. 5, 764-766; author reply 766 .

Sato, M., Vardy, L., Angel Garcia, M., Koonrugsa, N., and Toda, T. (2004). Interdependency of fission yeast Alp14/TOG and coiled coil protein Alp7 in microtubule localization and bipolar spindle formation. Mol. Biol. Cell 15, 1609-1622.

Schmidt, S., Essmann, F., Cirstea, I.C., Kuck, F., Thakur, H.C., Singh, M., Kletke, A., Jänicke, R.U., Wiek, C., Hanenberg, H., et al. (2010a). The centrosome and mitotic spindle apparatus in cancer and senescence. Cell Cycle 9, 4469-4473.

Schmidt, S., Schneider, L., Essmann, F., Cirstea, I.C., Kuck, F., Kletke, A., Jänicke, R.U., Wiek, C., Hanenberg, H., Ahmadian, et al. (2010b). The centrosomal protein TACC3 controls paclitaxel sensitivity by modulating a premature senescence program. Oncogene 29, 6184-6192.

Schneider, L., Essmann, F., Kletke, A., Rio, P., Hanenberg, H., Schulze-Osthoff, K., Nürnberg, B., and Piekorz, R.P. (2008). TACC3 depletion sensitizes to paclitaxel-induced cell death 
and overrides p21WAF-mediated cell cycle arrest. Oncogene 27, 116-125.

Schneider, L., Essmann, F., Kletke, A., Rio, P., Hanenberg, H., Wetzel, W., Schulze-Osthoff, K., Nürnberg, B., and Piekorz, R.P. (2007). The transforming acidic coiled coil 3 protein is essential for spindle-dependent chromosome alignment and mitotic survival. J. Biol. Chem. 282, 29273-29283.

Schuendeln, M.M., Piekorz, R.P., Wichmann, C., Lee, Y., McKinnon, P.J., Boyd, K., Takahashi, Y., and Ihle, J.N. (2004). The centrosomal, putative tumor suppressor protein TACC2 is dispensable for normal development, and deficiency does not lead to cancer. Mol. Cell. Biol. 24, 6403-6409.

Sillje, H.H., Nagel, S., Korner, R., and Nigg, E.A. (2006). HURP is a Ran-importin beta-regulated protein that stabilizes kinetochore microtubules in the vicinity of chromosomes. Curr. Biol. 16, 731-742.

Singh, D., Chan, J.M., Zoppoli, P., Niola, F., Sullivan, R., Castano, A., Liu, E.M., Reichel, J., Porrati, P., Pellegatta, S., et al. (2012). Transforming fusions of FGFR and TACC genes in human glioblastoma. Science 337, 1231-1235.

Srayko, M., Quintin, S., Schwager, A., and Hyman, A.A. (2003). Caenorhabditis elegans TAC- 1 and ZYG- 9 form a complex that is essential for long astral and spindle microtubules. Curr. Biol. 13, 1506-1511.

Still, I., Vettaikkorumakankauv, A., DiMatteo, A., and Liang, P. (2004). Structure-function evolution of the transforming acidic coiled coil genes revealed by analysis of phylogenetically diverse organisms. BMC Evol. Biol. 4, 16.

Still, I.H., Hamilton, M., Vince, P., Wolfman, A., and Cowell, J.K. (1999a). Cloning of TACC1, an embryonically expressed, potentially transforming coiled coil containing gene, from the 8 p11 breast cancer amplicon. Oncogene 18, 4032-4038.

Still, I.H., Vince, P., and Cowell, J.K. (1999b). The third member of the transforming acidic coiled coil-containing gene family, TACC3, maps in 4p16, close to translocation breakpoints in multiple myeloma, and is upregulated in various cancer cell lines. Genomics 58, 165-170.

Takayama, K., Horie-Inoue, K., Suzuki, T., Urano, T., Ikeda, K., Fujimura, T., Takahashi, S., Homma, Y., Ouchi, Y., and Inoue, S. (2012). TACC2 is an androgen-responsive cell cycle regulator promoting androgen-mediated and castration-resistant growth of prostate cancer. Mol. Endocrinol. 26, 748-761.
Tan, S., Lyulcheva, E., Dean, J., and Bennett, D. (2008). Mars promotes dTACC dephosphorylation on mitotic spindles to ensure spindle stability. J. Cell Biol. 182, 27-33.

Thakur, H.C. (2012). Biochemical and biophysical characterization of the centrosomal protein TACC3. Dissertation (Faculty of Mathematics and Natural Sciences of the Heinrich Heine University, Düsseldorf, Germany), November 2012.

Tsou, A.P., Yang, C.W., Huang, C.Y., Yu, R.C., Lee, Y.C., Chang, C.W., Chen, B.R., Chung, Y.F., Fann, M.J., Chi, C.W., et al. (2003). Identification of a novel cell cycle regulated gene, HURP, overexpressed in human hepatocellular carcinoma. Oncogene 22, 298-307.

Widlund, P.O., Stear, J.H., Pozniakovsky, A., Zanic, M., Reber, S., Brouhard, G.J., Hyman, A.A., and Howard, J. (2011). XMAP215 polymerase activity is built by combining multiple tubulinbinding TOG domains and a basic lattice-binding region. Proc. Natl. Acad. Sci. USA 108, 2741-2746.

Williams, S.V., Hurst, C.D., and Knowles, M.A. (2012). Oncogenic FGFR3 gene fusions in bladder cancer. Hum. Mol. Genet. 22, 795-803.

Wong, J., Lerrigo, R., Jang, C.Y., and Fang, G. (2008). Aurora A regulates the activity of HURP by controlling the accessibility of its microtubule-binding domain. Mol. Biol. Cell 19, 2083-2091.

Wurdak, H., Zhu, S., Min, K.H., Aimone, L., Lairson, L.L., Watson, J., Chopiuk, G., Demas, J., Charette, B., Halder, R., et al. (2010). A small molecule accelerates neuronal differentiation in the adult rat. Proc. Natl. Acad. Sci. USA 107, 16542-16547.

Yao, R., Natsume, Y., and Noda, T. (2007). TACC3 is required for the proper mitosis of sclerotome mesenchymal cells during formation of the axial skeleton. Cancer Sci. 98, 555-562.

Yao, R., Natsume, Y., Saiki, Y., Shioya, H., Takeuchi, K., Yamori, T., Toki, H., Aoki, I., Saga, T., and Noda, T. (2011). Disruption of Tacc3 function leads to in vivo tumor regression. Oncogene 31, 135-148.

Yu, C.T., Hsu, J.M., Lee, Y.C., Tsou, A.P., Chou, C.K., and Huang, C.Y. (2005). Phosphorylation and stabilization of HURP by Aurora-A: implication of HURP as a transforming target of Aurora-A. Mol. Cell Biol. 25, 5789-5800.

Zyss, D. and Gergely, F. (2009). Centrosome function in cancer: guilty or innocent? Trends Cell Biol. 19, 334-346. 